\title{
Automated functional upper limb evaluation of patients with Friedreich ataxia using serious games rehabilitation exercises
}

Bruno Bonnechère ${ }^{1,2,3^{*}} \mathbb{D}$, Bart Jansen ${ }^{2,3}$, Inès Haack', Lubos Omelina ${ }^{2,3}$, Véronique Feipel ${ }^{4}$, Serge Van Sint Jan ${ }^{1}$ and Massimo Pandolfo ${ }^{5}$

\begin{abstract}
Background: Friedreich ataxia (FRDA) is a disease with neurological and systemic involvement. Clinical assessment tools commonly used for FRDA become less effective in evaluating decay in patients with advanced FRDA, particularly when they are in a wheelchair. Further motor worsening mainly impairs upper limb function. In this study, we tested if serious games (SG) developed for rehabilitation can be used as an assessment tool for upper limb function even in patients with advanced FRDA.

Methods: A specific SG has been developed for physical rehabilitation of patients suffering from neurologic diseases. The use of this SG, coupled with Kinect sensor, has been validated to perform functional evaluation of the upper limbs with healthy subjects across lifespan. Twenty-seven FRDA patients were included in the study. Patients were invited to perform upper limb rehabilitation exercises embedded in SG. Motions were recorded by the Kinect and clinically relevant parameters were extracted from the collected motions. We tested if the existence of correlations between the scores from the serious games and the severity of the disease using clinical assessment tools commonly used for FRDA. Results of patients were compared with a group a healthy subjects of similar age.

Results: Very highly significant differences were found for time required to perform the exercise (increase of $76 \%$, $\mathrm{t}(68)=7.22, P<0.001$ ) and for accuracy (decrease of $6 \%, \mathrm{t}(68)=-3.69, P<0.001$ ) between patients and healthy subjects. Concerning the patients significant correlations were found between age and time $(R=0.65, p=0.015)$, accuracy $(R=-0.75, p=0.004)$ and the total displacement of upper limbs. $(R=0.55, p=0.031)$. Statistically significant correlations were found between the age of diagnosis and speed related parameters.

Conclusions: The results of this study indicate that SG reliably captures motor impairment of FRDA patients due to cerebellar and pyramidal involvement. Results also show that functional evaluation of FRDA patients can be performed during rehabilitation therapy embedded in games with the patient seated in a wheelchair.
\end{abstract}

Trial registration: The study was approved as a component of the EFACTS study (Clinicaltrials.gov identifier NCT02069509, registered May 2010) by the local institutional Ethics Committee (ref. P2010/132).

Keywords: Serious games, Assessment, Evaluation, Friedreich Ataxia, Kinect sensor

\footnotetext{
*Correspondence: bbonnech@ulb.ac.be

'Laboratory of Anatomy, Biomechanics and Organogenesis (LABO) [CP 619],

Université Libre de Bruxelles, Lennik Street 808, 1070 Brussels, Belgium

2Department of Electronics and Informatics - ETRO, Vrije Universiteit Brussel,

Brussels, Belgium

Full list of author information is available at the end of the article
}

(c) The Author(s). 2018 Open Access This article is distributed under the terms of the Creative Commons Attribution 4.0 International License (http://creativecommons.org/licenses/by/4.0/), which permits unrestricted use, distribution, and reproduction in any medium, provided you give appropriate credit to the original author(s) and the source, provide a link to the Creative Commons license, and indicate if changes were made. The Creative Commons Public Domain Dedication waiver (http://creativecommons.org/publicdomain/zero/1.0/) applies to the data made available in this article, unless otherwise stated. 


\section{Background}

Friedreich ataxia (FRDA) is an autosomal recessive disease with neurological and systemic involvement. Most commonly, loss of balance in a child or adolescent is the first symptom. Within a few years, trunk and limb ataxia become prominent, accompanied by dysarthria, oculomotor abnormalities and weakness. At the onset of disease, ataxia is mostly due to loss of large proprioceptive primary sensory neurons in the dorsal root ganglia (DRGs), with associated atrophy of the posterior columns and spinocerebellar tracts. With progression, cerebellar ataxia, due to atrophy of the dentate nucleus, becomes prevalent. Also with progression, distal axonal loss in the pyramidal tracts that cause further motor impairment $[1,2]$. Most affected individuals become unable to walk within $10-15$ years since disease onset, but then continue to deteriorate because of worsening upper limb ataxia, weakness, dysarthria, and eventually dysphagia. Clinical assessment tools commonly used for FRDA, such as the Scale for Assessment and Rating of Ataxia (SARA) [3], the Friedreich Ataxia Rating Scale (FARS) [4], and the International Cooperative Ataxia Rating Scale (ICARS) [5], become less effective in evaluating progression in patients with advanced FRDA, particularly when they are in a wheelchair and when upper limb function is impaired. This is partly due to a ceiling effect in these scales, in which gait and balance item have a major weight, but also to the characteristics of the disease $[6,7]$. In FRDA, pyramidal symptoms like slowing and impairment of rapid alternating movements and difficulties in raising arms to point a target, eventually greatly affect performance in upper limb coordination tests, making their scoring more erratic. Hence the need for a more integrated tool to assess upper limb motor function in FRDA, particularly in wheelchair-bound patients with advanced disease.

Commercial video games have significantly evolved over the last decade. Today computer performance and play experience allow new perspectives for rehabilitation. Thanks to new gaming controllers (Nintendo Wii FitTM, Microsoft Xbox KinectTM, etc.) video game playing has changed from a passive (e.g., the player is seated on a sofa using a very simple controller) to an active experience: players have to move in order to interact with games (e.g., the player is truly active with a game controller requesting full body movements). Clinicians are now prospecting the new potential use of these games in rehabilitation mainly through testing available commercial games with patients suffering from various neurological pathologies (e.g. Cerebral Palsy [8], stroke [9], Parkinson disease [10]). Although encouraging results have been observed, especially in terms of motivation, there are several problems related to the use of commercial video games in rehabilitation. The player motion accuracy requested from the player during the games is low while most therapists will aim to improve patient joint control and coordination and the motion that must be performed to control the games do not correspond to rehabilitation exercises. Furthermore, there is currently no possibility to record the motion performed by the patients during those kind of exercises. However, collecting this information could be important to: (i) allow to provide direct feedback to patient and eventually correct the motion if they are not performed in the right way and (ii) to provide information to therapists in case of telerehabilitation exercises when the patient is performing exercises at home without the clinicians' supervision [11].

In order to tackle the above-mentioned limitations, specific games must be developed for rehabilitation purposes [12]. Such kind of games, called serious games (SG) (i.e. games designed with a primary purpose other than pure entertainment), must be designed taking into account real clinical needs and constraints (e.g. simple visual background, based on relevant clinical schemes, range of motion and speed required to perform the exercises must be adaptable, etc.) [13] and allow to record motions performed by the patients $[14,15]$.

Depending on the joints and the kind of motions performed, results obtained with the Kinect sensor must be analyzed carefully. However, it was shown that using the displacement of wrist relative to trunk appears to be a good approach to evaluate upper limb function during rehabilitation exercises using serious games [15]. Furthermore, results obtained from Kinect and gold-standard optoelectronic device to assess upper limb functions are significantly correlated [16].

In this study, we tested if SG can be used as an assessment tool for upper limb function in patients with advanced FRDA. First, we determined if the scores obtained in the SG are able to differentiate patients and healthy participants. Then, we correlated SG results with genetic and clinical parameters of disease severity. To the authors best knowledge, there is currently no study about the use of SG to perform functional evaluation of patients with FRDA simultaneously with physical rehabilitation exercises.

\section{Methods \\ Patients}

Twenty-seven patients were included in the study, characteristics of the patients are presented in Table 1. They were all enrolled in the European Friedreich Ataxia Consortium for Translational Studies (EFACTS, Clinicaltrials.gov identifier NCT02069509) natural history study, the first prospective pan-European FRDA registry designed to define clinical rating scales and quality of life measures, which can be used in clinical trials. Since its inception in 2010, EFACTS has enrolled more than 800 genetically confirmed FRDA patients in nine European countries. SG was added to the EFACTS follow-up protocol at the Brussels site as an additional functional assessment. Data presented here are cross-sectional, for each patient they have been obtained at 
Table 1 Characteristics of the patients included in the study

\begin{tabular}{lll}
\hline Variables & $\mathrm{N}$ & Mean (std) \\
\hline Age (years) & 27 & $26,0(12.2)$ \\
Duration of the disease (years) & 27 & $15.0(7,44)$ \\
SARA score & 27 & $22,5(9,2)$ \\
ADL score & 27 & $16,9(6.7)$ \\
GAA-1 number of repeats & 15 & $608.2(306.4)$ \\
9 R 1 (s) & 25 & $72.01(42.3)$ \\
9 R 2 (s) & 25 & $68.1(41.6)$ \\
9 L 1 (s) & 25 & $87.2(57.1)$ \\
9 L 2 (s) & 25 & $79.3(47.0)$ \\
CCFS F score (with writing) & 25 & $1.33(0.37)$ \\
CCFS H score (without writing) & 25 & $1.31(0.37)$ \\
\hline
\end{tabular}

time of a single annual EFACTS visit. The study was approved by the local institutional Ethics Committee (ref. P2010/132) and patients gave written informed consent.

A database of healthy subjects ranging from 5 to 90 years was created in a previous study with the aim of using these data for comparison with patients by selecting the appropriate age range [15]. Exclusion criteria for the healthy subjects were neurological conditions, balance deficits or orthopedic disorders in the last six months. Forty-three healthy subjects have been used as an age-matched control group (26 (11) years old, 20 women).

\section{Clinical evaluation}

The clinical assessment of EFACTS patients includes structured interviews, questionnaires, performance-based coordination tests, clinical disease rating scales and neuropsychological functional measures. Sampling of biomaterials and genetic analysis complement the examination. GAA repeat lengths for both alleles (GAA-1 and GAA-2) are determined at the ULB Laboratory of Experimental Neurology [17] Table 1.

Details of the clinical assessment have been previously reported [6]. Briefly, clinical tests whose scores have been correlated with those obtained from the SG include the Scale for the Assessment and Rating of Ataxia (SARA) [3], which quantifies ataxia symptoms based on eight items with a maximum score of 40; the Inventory of Non-Ataxia Symptoms (INAS) [18], which provides a checklist of non-ataxia symptoms such as changes in reflexes, other motor and sensory symptoms, ophthalmological findings, urinary dysfunction, and cognitive impairment; the Spinocerebellar Ataxia Functional Index (SCAFI), consisting of timed performance measures including an $8 \mathrm{~m}$ walk at maximum speed, the nine-hole peg test, and the rate of repeating the syllables "PATA" within 10s; the Cerebellar Composite Functional Score (CCFS) [19], a computerized set of timed tests of upper limb coordination performed with the dominant hand that includes a rapidly alternating click test, the 9-hole peg test and an optional writing test, which generates a composite score (higher values correspond to more impairment) including (F) or excluding $(\mathrm{H})$ the optional writing test; the functional staging for ataxia part of the Friedreich Ataxia Rating Scale (FARS) [4]; the basic activities of daily living (ADL) part of the FARS was used to assess impairment in the ability to perform daily life activities.

\section{The serious game}

Participants played one mini-game specially developed for physical rehabilitation as part of a previous project (called ICT4Rehab), the Wipe Out game [13]. The player has to clean the screen virtually covered by some virtual fog using a tissue controlled by medio-lateral and inferior-superior displacements of the upper limb (wrist) relative to the trunk (Fig. 1). Spatial displacements of the players were recorded by a Kinect sensor [14]. Motion data were stored in standard format (i.e., C3D) for further analysis. Participants were invited to stand in front of the screen and the sensor. Patients were asked to play the games three times. The mean of the three repetitions was used for statistical analysis. This was similar to previously published functional evaluation protocols using SG [15].

\section{Data processing}

The use of this SG has been validated to perform functional evaluation of the upper limbs with healthy subjects across lifespan [15]. Two parameters were processed from the games: the time required to clean $90 \%$ of the screen and the accuracy. The accuracy of the motion was assessed by computing the number of times that the subject is placing the cloth in the same position on the screen (Eq.1). The number of frames where the cloth was in a position that had already been cleaned were computed ("cleaned" frames). The result was finally expressed in percentage. The higher the accuracy score, the better the results in term of performance.

$$
\text { accuracy }=100-\left(\left(\frac{\text { Number of }{ }^{\prime \prime} \text { cleaned" frames }}{\text { Total number of frames }}\right) \times 100\right)
$$

Several parameters were then processed from the motion performed by the patients and recorded by the Kinect, those parameters are adapted from previous studies on the validation of scores to assess dynamic balance during rehabilitation exercises [20]: the total displacement of the wrist related to the trunk (DOT) based on medio-lateral (ML) and top-down (TD) displacements (Eq. 2), the area of 95\% confidence prediction ellipse (Area) (Eq. 3), the dispersion of the trajectory from the mean position in ML and TD directions $\left(\mathrm{RMS}_{\mathrm{ML}}\right.$ and $\mathrm{RMS}$ TD) (Eqs. 4 and 5), the 

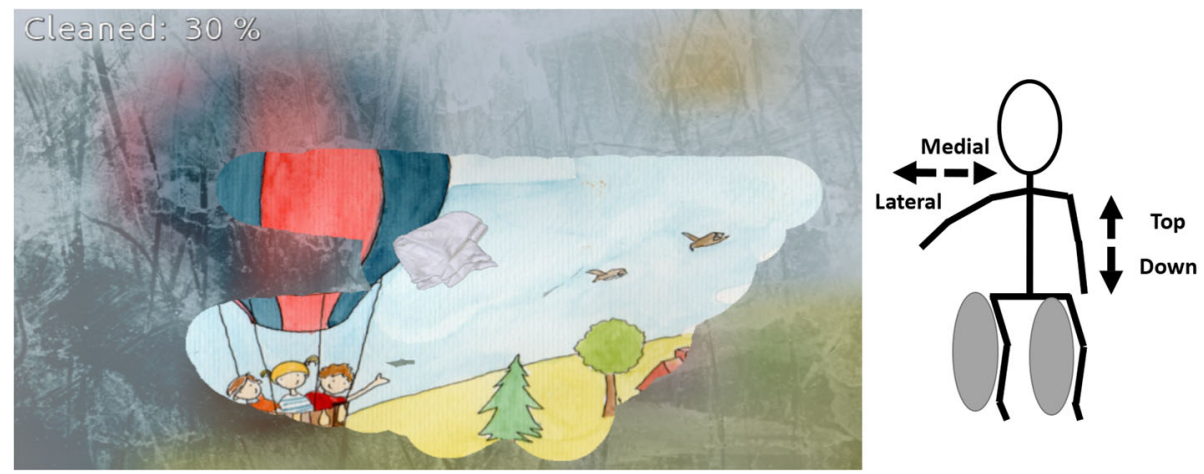

Fig. 1 Screenshot of the game

range of displacement $\left(\mathrm{R}_{\mathrm{ML}}\right.$ and $\mathrm{R}_{\mathrm{TD}}$ (Eqs. 6 and 7), the mean velocity displacement $\left(\mathrm{MV}_{\mathrm{ML}}\right.$ and $\left.\mathrm{MV}_{\mathrm{TD}}\right)$ (Eqs. 8 and 9) and the total mean velocity (TMV) (Eq. 10).

$$
\begin{aligned}
& D O T=\sum_{i=1}^{N} \sqrt{M L(i)^{2}+T D(i)^{2}} \\
& \text { Area }=\pi \times \operatorname{prod}(2.4478 \times \sqrt{\operatorname{svd}(\operatorname{eig}(\operatorname{cov}(M L, T D)))} \\
& R_{M S}=\frac{1}{N} \sqrt{\sum_{i=1}^{N} M L(i)^{2}} \\
& R_{M S}=\frac{1}{N} \sqrt{\sum_{i=1}^{N} T D(i)^{2}} \\
& R_{M L}=\max (M L)-\min (M L) \\
& R_{T D}=\max (T D)-\min (T D) \\
& M V_{M L}=\frac{f}{N} \sum_{i=1}^{N-1}|M L(i+1)-M L(i)| \\
& M V_{T D}=\frac{f}{N} \sum_{i=1}^{N-1}|T D(i+1)-T D(i)| \\
& T M V=\frac{f}{N} \sum_{i=1}^{N-1}{\sqrt{(M L(i+1)-M L(i))^{2}+(T D(i+1)-T D(i))^{2}}}^{2}
\end{aligned}
$$

Data were processed and plotted using a customized software routine developed in Matlab 2017.

\section{Statistics}

To detect a difference of $40 \%$ of the time required to perform the exercise between patients and control with $80 \%$ power and a two-sided type I error of $5 \%$, we calculated that we need to include 24 patients.

Normality of each parameter was checked using the Shapiro-Wilk tests and by graphical analysis (histogram, boxplot and normal Q-Q plot). Pearson's correlation coefficients were used to determine if the scores from the SG were correlated with the clinical evaluation. Independent sample T-test were applied to compare patients and healthy subjects. Statistics were performed with SPSS 20 and RStudio (version 1.1.442) with $\mathrm{R}$ version 3.4.4, significance level was set at 0.05 .

\section{Results}

Age is an important factor for both gross and fine motor control [21, 22] and since the range of age is important in our group of patients (from 6 to 48 years old) results for time and accuracy were plotted according to the age of the participants and compared to results of the healthy control group in Figs. 2 and 3 respectively. Quadratic fitting with 95\% CI were plotted since it has been previously demonstrated that this fitting was the most adapted for this kind of data [15]. Very highly significant differences were found for time (increased of $76 \%, \mathrm{t}(68)=7.22, P<0.001$ ) and for accuracy (decreased of $6 \%, \mathrm{t}(68)=-3.69, P<0.001)$ between healthy subjects and patients. Results of all the studied parameters are presented in Table 2. Since no significant differences were found between right and left side for any of the parameters mean values were presented.

Pearson's correlation coefficients are presented in Table 3. Statistically significant correlations were found between age and Time $(R=0.65, p=0.015)$, accuracy $(R=-0.75, p=0.004)$ and the total displacement of upper limbs (DOT) $(R=0.55, p=0.031)$. Statistically significant correlations were found between the age of diagnosis and the speed related parameters $(R=-0.53, p=0.021$ and $R=-0.49, p=0.029$ for $M V_{M L}$ and $M V_{\text {IS }}$ respectively).

Statistically significant correlations were found for TD displacements ( $R=0.48$ and 0.47 for ranges and RMS) and the basic activities of daily living.

Correlations were found between the nine-hole peg tests and the DOT $(R=0.60, p=0.012)$, the area $(R=$ $0.59, p=0.025)$, the amplitudes $(R=0.45, p=0.041$ and $R=0.49, p=0.033$ for ML and TD displacements respectively). For the speed correlations were found only for the TD direction $(R=0.43, p=0.030)$ not for ML. 


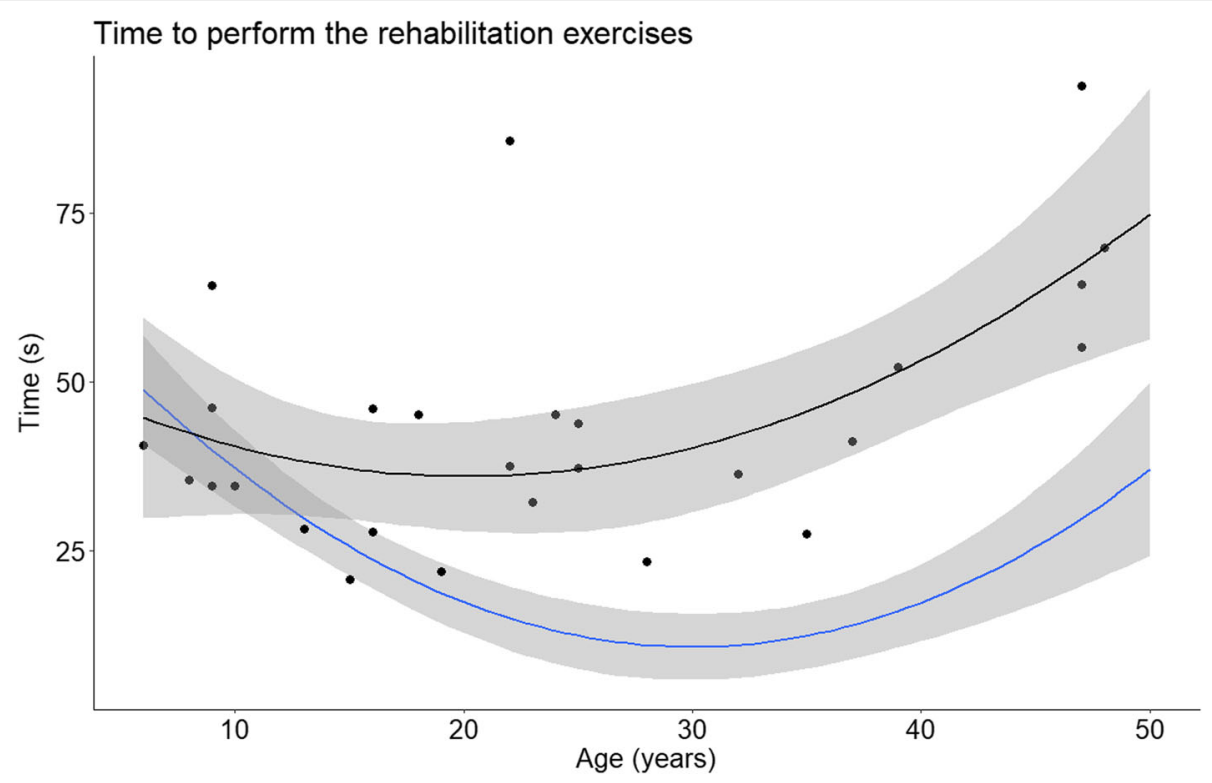

Fig. 2 Mean and 95\% confidence interval (Cl) of the time for healthy subjects (blue) and patient results (black). Black dots represent individual results of the patients

Concerning the comparison with the genetic analysis statistically significant correlations were found between the parameters extracted from the SG (DOT, $\mathrm{RMS}_{\mathrm{ML}}$, $\mathrm{RMS}_{\mathrm{TD}}$ and $\mathrm{R}_{\mathrm{ML}}$ ) and GAA-1.

\section{Discussion}

During the course of FRDA, patients gradually become unable to stand and walk. Therefore, it is essential that an upper limb coordination test like SG can be performed with the patient seated in a wheelchair. Although previous studies reported some limitations of the Kinect sensor to measure upper limb joint angles of patients in wheelchairs [23], this study demonstrates it is feasible to combine rehabilitation exercises and functional evaluation of FRDA patients, even the more disabled ones.

The first aim of this study was to determine if functional analysis performed with the SG can be used to differentiate

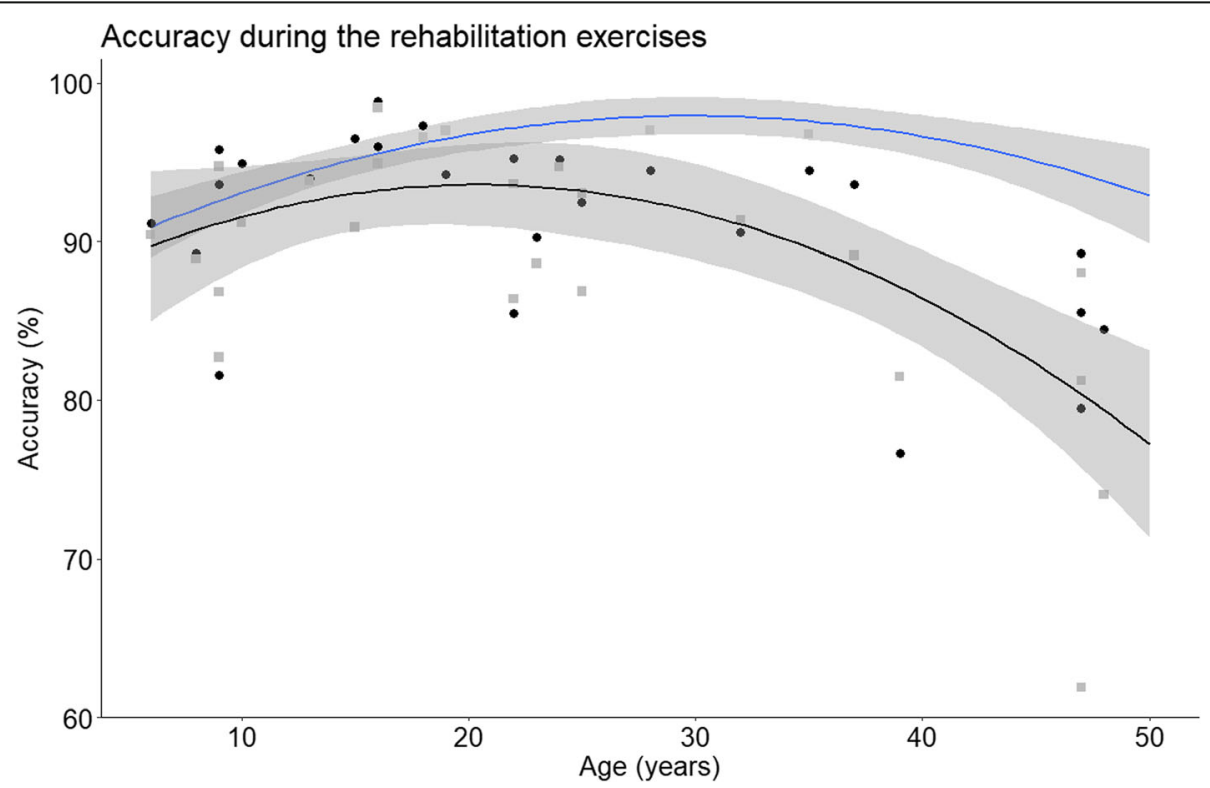

Fig. 3 Mean and 95\% Cl of the accuracy for healthy subjects (blue) and patient results (black). Black dots (right side) and grey squares (left side) represent individual results of the patients. Since no statistically significant difference were found between right and left side mean of the two sides was used for the fitting 
Table 2 Mean (std) results for patients and control

\begin{tabular}{|c|c|c|c|c|}
\hline Variables & Patients $(n=27)$ & Control $(n=43)$ & T-test & Difference [95\% Cl] \\
\hline Time (s) & $44(18)$ & $25(15)$ & $\mathrm{t}(68)=7.22, P<0.001$ & $19[12 ; 29]$ \\
\hline Accuracy (\%) & $88(7)$ & $94(6)$ & $\mathrm{t}(68)=3.69, P<0.001$ & $6[3 ; 9]$ \\
\hline DOT (cm) & $1462(664)$ & $1150(350)$ & $\mathrm{t}(68)=2.24, P=0.026$ & $312[40 ; 583]$ \\
\hline Area $\left(\mathrm{cm}^{2}\right)$ & $1421(913)$ & $1278(576)$ & $\mathrm{t}(68)=0.74, P=0.458$ & $143[-242 ; 528]$ \\
\hline $\mathrm{RMS}_{\mathrm{ML}}(\mathrm{cm})$ & $10(3)$ & $8(4)$ & $\mathrm{t}(68)=2.38, P=0.018$ & $2[0.3 ; 3.7]$ \\
\hline $\mathrm{RMS}_{\mathrm{TD}}(\mathrm{cm})$ & $7(3)$ & $5(2)$ & $\mathrm{t}(68)=3.06, P=0.003$ & $2[0.7 ; 3.3]$ \\
\hline $\mathrm{R}_{\mathrm{ML}}(\mathrm{cm})$ & $55(11)$ & $48(8)$ & $\mathrm{t}(68)=2.86, P=0.005$ & $7[2.2 ; 11.8]$ \\
\hline $\mathrm{R}_{\mathrm{TD}}(\mathrm{cm})$ & $44(10)$ & $39(7)$ & $\mathrm{t}(68)=2.27, P=0.024$ & $5[0.7 ; 9.3]$ \\
\hline $\mathrm{MV}_{\mathrm{ML}}(\mathrm{cm} / \mathrm{s})$ & $38(16)$ & $56(10)$ & $\mathrm{t}(68)=5.23, P<0.001$ & $18[13 ; 24]$ \\
\hline $\mathrm{MV}_{\mathrm{TD}}(\mathrm{cm} / \mathrm{s})$ & $33(14)$ & $53(9)$ & $\mathrm{t}(68)=6.61, P<0.001$ & $20[14 ; 26]$ \\
\hline $\mathrm{TMV}(\mathrm{cm} / \mathrm{s})$ & $57(20)$ & $81(10)$ & $\mathrm{t}(68)=5.19, P<0.001$ & $26[16 ; 32]$ \\
\hline
\end{tabular}

$P$-value is the results of T-tests

patient and healthy subjects. Statistically significant differences were obtained for most of the studied parameters except for the area $(p=0.37)$ indicating that the patients stay within an acceptable range in the limits of the screen. By separately analyzing the two components (medio-lateral and top-down) of motion we observed that the difference is due to a too large amplitude in the ML direction whereas there is no difference at the TD level.

Figures 2 and 3 show that until the age of 20 patients seem to remain in the confidence interval of the control group, differences only appeared later in the course of the disease. Similar trends were found for all parameters. In order to better visualize this phenomenon, results were normalized and expressed as a percentage of control group values. In Fig. 4 results are plotted according to disease duration. There was a statistically significant correlation between disease duration and decreased speed
( $R=0,64, p=0,002)$. Interestingly, the decrease in speed was associated with a significant reduction of accuracy ( $R=-0,67, p=0,001)$. We also analyzed the relation between speed and accuracy. For FRDA there is a higher correlation $(R=-0.87, p<0.001)$ than for healthy subjects $(R=-0.72, p<0.001)$. We divided the subjects according to age (bellow or above 20 years old) and plotted the results in Fig. 5. For participants aged less than 20 years the slope of the regression line was $\beta=-1.8, p=0.022, \mathrm{R}^{2}=$ 0.42 for FRDA and $\beta=-3.5, p<0.001, R^{2}=0.54$ for healthy subjects. For participants older than 20 years the slope of the regression line is: $\beta=-2.3, p<0.001, \mathrm{R}^{2}=$ 0.68 for FRDA and $\beta=-1.4, p<0.001, \mathrm{R}^{2}=0.46$ for healthy subjects.

These results indicate that SG reliably captures motor impairment of FRDA patients due to cerebellar and pyramidal involvement. Results also show that functional

Table 3 Pearson's correlation coefficients (R) between scores obtained from the SG and the clinical evaluation

\begin{tabular}{|c|c|c|c|c|c|c|c|c|c|c|c|}
\hline Variables & Time & Accuracy & DOT & Area & RMS $_{\mathrm{ML}}$ & $\mathrm{RMS}_{\mathrm{TD}}$ & $\mathrm{R}_{\mathrm{ML}}$ & $\mathrm{R}_{\mathrm{TD}}$ & $\mathrm{MV} \mathrm{ML}_{\mathrm{L}}$ & $\mathrm{MV}_{\mathrm{TD}}$ & TMV \\
\hline Age (year) & $.63^{* *}$ & $-.73^{* *}$ & $.57^{*}$ & -.16 & -0.04 & -.20 & -.06 & -.20 & -.16 & -.20 & -.41 \\
\hline Age of diagnosis (year) & .44 & $-.64^{* *}$ & .28 & -.10 & -.23 & -.12 & -.18 & -.36 & $-.53^{*}$ & $-.49 *$ & -.19 \\
\hline Duration of the disease (year) & $.64^{* *}$ & $-.67^{* *}$ & $.48^{* *}$ & -.25 & .26 & .26 & .32 & .06 & -.16 & -.25 & -.25 \\
\hline SARA score & .27 & -.21 & $.46^{*}$ & .32 & .35 & .24 & .32 & .29 & .25 & .21 & .21 \\
\hline ADL score & .13 & .15 & .38 & .37 & $.50^{*}$ & $.59^{*}$ & $.39 *$ & $.50 *$ & .36 & .28 & .28 \\
\hline GAA-1 number of repeats & -.18 & .28 & $.61 *$ & .39 & $.47^{*}$ & $.58^{*}$ & $.64^{* *}$ & .39 & .26 & .01 & .11 \\
\hline 9 R 1 (s) & .14 & .22 & $.61 *$ & $.64^{*}$ & .01 & $.59^{*}$ & $.50 *$ & $.49^{*}$ & $.38^{*}$ & $.46^{*}$ & $.41 *$ \\
\hline 9 R 2 (s) & .15 & .0 .20 & $.61 *$ & $.63^{*}$ & -.02 & $.55^{*}$ & $.48^{*}$ & $.50 *$ & .37 & $.46^{*}$ & $.41 *$ \\
\hline $9\llcorner 1(\mathrm{~s})$ & .18 & $.65^{* *}$ & $.61^{*}$ & $.51 *$ & .36 & $.59 *$ & .41 & $.48^{*}$ & .30 & $.40^{*}$ & .32 \\
\hline $9\llcorner 2(s)$ & -.16 & 0.11 & $.63^{*}$ & $.60^{*}$ & .12 & $.54^{*}$ & $.43^{*}$ & $.49^{*}$ & .32 & $.41 *$ & .24 \\
\hline CCFS F score (with writing) & -.13 & .29 & .16 & .36 & -.13 & .15 & $-.43^{*}$ & .18 & $.47^{*}$ & .37 & $.44^{*}$ \\
\hline CCFS H score (without writing) & -.18 & .37 & .11 & .33 & .23 & .26 & .31 & .19 & .30 & .27 & .30 \\
\hline
\end{tabular}

*Significant correlation $(P<.05)$

**Significant correlation $(P<.01)$ 

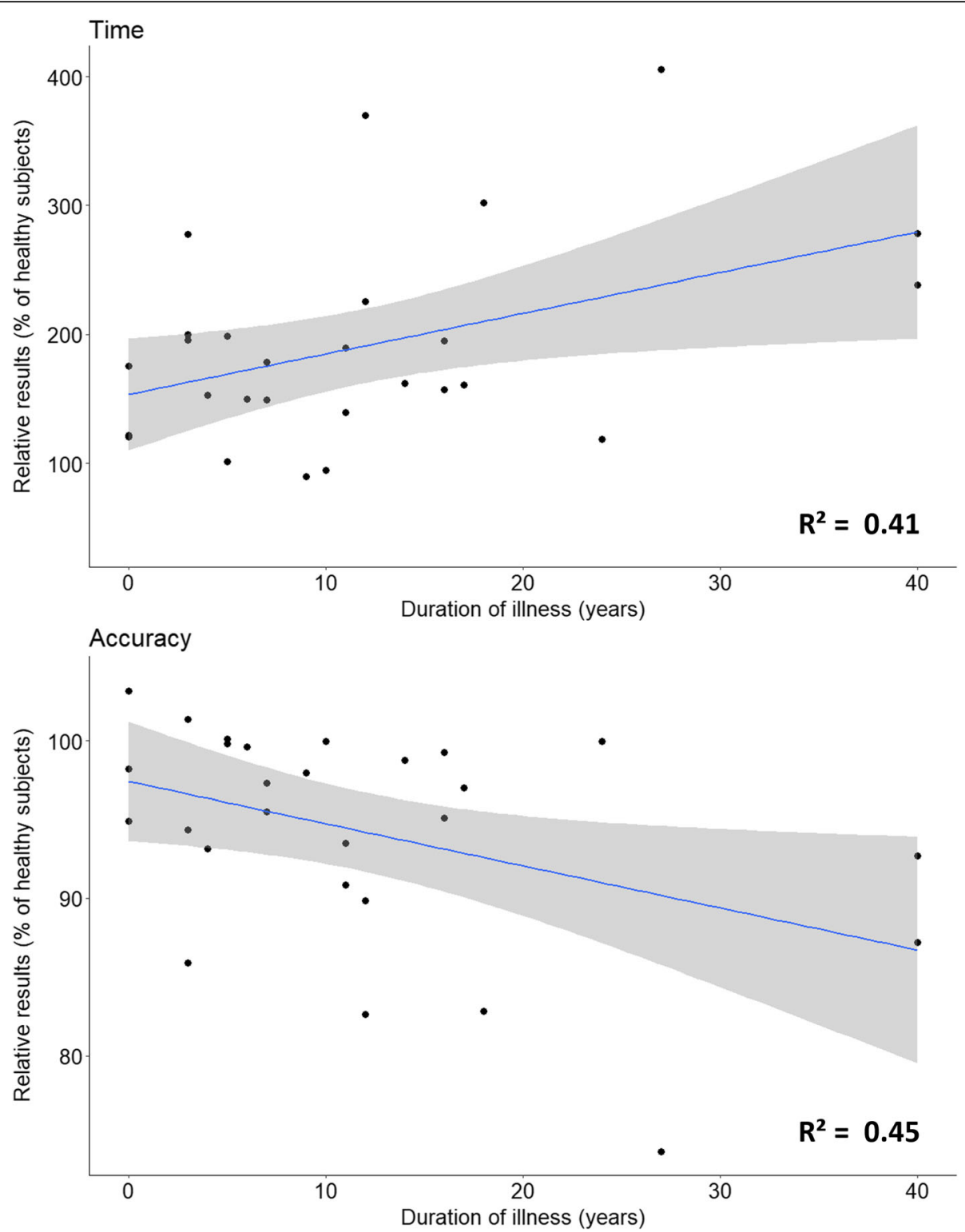

Fig. 4 Results of the time and accuracy expressed in percentage of the values of healthy subjects according to the duration of the disease. Linear fitting with $95 \% \mathrm{Cl}$ is presented with $\mathrm{R}^{2}$

evaluation of FRDA patients can be performed during rehabilitation therapy embedded in games. Younger patients who still perform within the confidence interval of the control group are likely to have a mainly afferent ataxia due to DRG pathology, so that it is probable that their motor performance under visual control is not degraded. The fact that GAA-1 correlates with several SG parameters is in agreement with other observations that the severity of the genetic mutation not only affects the age of symptom onset, but also the subsequent rate of progression and severity of symptoms [7]. Analysis of a larger sample and prospective follow-up of patients will assess the value of SG as a potential outcome measure for clinical trials, particularly in more advanced patients for which current evaluation tools becomes less effective with the evolution of the disease.

One limitation of this study is that only one session of measurement has been done, because patients only come once a year to the hospital for the EFACTS follow-up. Therefore, longitudinal studies are needed to determine the reproducibility of measurements and the influence of training, habituation or fatigue on the results.

Finally, the last point is to integrate these solutions into patients' home to motivate them to perform the rehabilitation exercises. In addition to this aspect of 
Relation time and accuracy

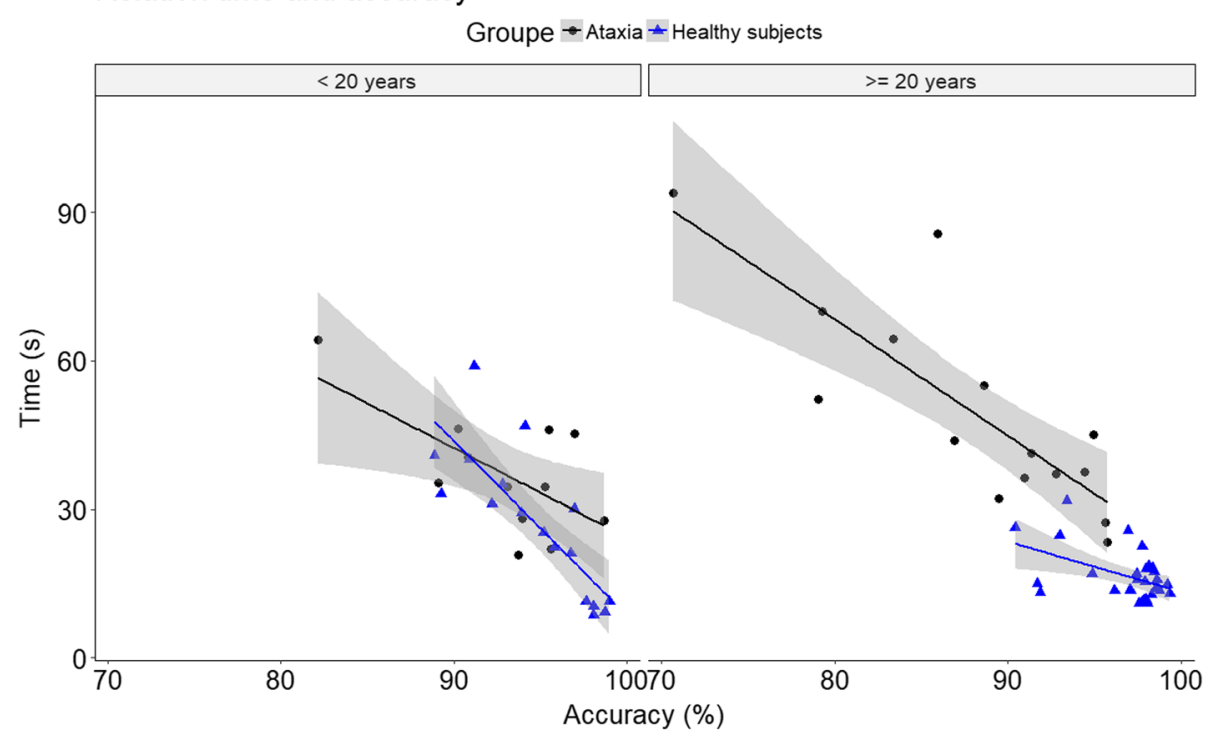

Fig. 5 Scatter plots and linear regression for the relation between time and accuracy according to age

motivation these kinds of solutions allow and evaluation of the quality of the rehabilitation exercise and thus a longitudinal follow-up for both patients and clinicians.

\section{Conclusion}

The use of new technologies in rehabilitation, including SG, is becoming increasingly important. In this study demonstrated that it is possible to combine rehabilitation exercises using SG and automated upper limb functional assessment in FRDA patients in wheelchairs. Future works are needed to determine if such kind of solution can be successfully integrated in the rehabilitation program and whether the kind of data presented in this paper can be used to predict disease progression.

\begin{abstract}
Abbreviations
ADL: Activity of daily living; DOT: Total displacement of the wrist related to the trunk; DRGs: Dorsal root ganglia; EFACTS: European Friedreich Ataxia Consortium for Translational Studies; FARS: Friedreich Ataxia Rating Scale; FRDA: Friedreich ataxia; ICARS: International Cooperative Ataxia Rating Scale; ML: Medio-lateral; MV: Mean velocity; R: Range; RMS: Dispersion of the trajectory from the mean position; SARA: Scale for Assessment and Rating of Ataxia; SG: Serious games; TD: Top-down; TMV: Total mean velocity
\end{abstract}

\section{Acknowledgements}

We extend our deepest thanks to all the participants who participated in this study. Thanks to Mrs. Alaerts and Fadel for their technical assistance.

\section{Funding}

This study is a part of the ICT4Rehab and RehabGoesHome projects (www.ict4rehab.org). Those projects were funded by Innoviris (Brussels Capital Region).

\section{Availability of data and materials}

The clinical datasets generated and/or analysed during the current study are not publicly available due to the property of EFACTS but data from the rehabilitation exercises are available from the corresponding author on request.
Authors' contributions

$B B, B J$ and SVSJ were responsible for the conception and design of the study. MP performed clinical investigations and acquired clinical data. BB and IH performed rehabilitation exercises using SG. The data were analyzed by $B B$ and VF. BB drafted the report and MP, LO, BJ, VF and SVSJ provided substantial input to the first draft. BB created the figures. All authors reviewed the manuscript and give final approval of the version to be submitted. The study was supervised by MP.

\section{Ethics approval and consent to participate}

All study participants gave their informed, written consent to participation, in line with Belgian ethical guidelines. The study was approved as a component of the EFACTS study by the local institutional Ethics Committee (ref. P2010/132).

\section{Competing interest}

The authors declares that they have no competing interest.

\section{Publisher's Note}

Springer Nature remains neutral with regard to jurisdictional claims in published maps and institutional affiliations.

\section{Author details}

'Laboratory of Anatomy, Biomechanics and Organogenesis (LABO) [CP 619], Université Libre de Bruxelles, Lennik Street 808, 1070 Brussels, Belgium. ${ }^{2}$ Department of Electronics and Informatics - ETRO, Vrije Universiteit Brussel, Brussels, Belgium. ${ }^{3}$ imec, Leuven, Belgium. ${ }^{4}$ Laboratory of Functional Anatomy (LAF), Université Libre de Bruxelles, Brussels, Belgium. ${ }^{5}$ Department of Neurology, Erasme Hospital, Brussels, Belgium.

Received: 26 April 2018 Accepted: 19 September 2018

Published online: 04 October 2018

\section{References}

1. Harding AE. Friedreich's ataxia: a clinical and genetic study of 90 families with an analysis of early diagnostic criteria and intrafamilial clustering of clinical features. Brain. 1981;104:589-620.

2. Koeppen AH. Friedreich's ataxia: pathology, pathogenesis, and molecular genetics. J Neurol Sci. 2011:303:1-12.

3. Schmitz-Hübsch T, Montcel du ST, Baliko L, Berciano J, Boesch S, Depondt C, et al. Scale for the assessment and rating of ataxia: development of a new clinical scale. Neurology. 2006;66:1717-20. 
4. Subramony SH, May W, Lynch D, Gomez C, Fischbeck K, Hallett M, et al. Measuring Friedreich ataxia: interrater reliability of a neurologic rating scale. Neurology. 2005;64:1261-2.

5. Metz G, Coppard N, Cooper JM, Delatycki MB, Dürr A, Di Prospero NA, et al. Rating disease progression of Friedreich's ataxia by the international cooperative Ataxia rating scale: analysis of a 603-patient database. Brain. 2013;136:259-68

6. Reetz K, Dogan I, Costa AS, Dafotakis M, Fedosov K, Giunti P, et al. Biological and clinical characteristics of the European Friedreich's Ataxia consortium for translational studies (EFACTS) cohort: a cross-sectional analysis of baseline data. Lancet Neurol. 2015:14:174-82.

7. Reetz K, Dogan I, Hilgers R-D, Giunti P, Mariotti C, Dürr A, et al. Progression characteristics of the European Friedreich's Ataxia consortium for translational studies (EFACTS): a 2 year cohort study. Lancet Neurol. 2016;15:1346-54.

8. Bonnechère $B$, Jansen $B$, Omelina $L$, Degelaen $M$, Wermenbol $V$, Rooze $M$, et al. Can serious games be incorporated with conventional treatment of children with cerebral palsy? A review. Res Dev Disabil. 2014;35:1899-913.

9. Putrino D. Telerehabilitation and emerging virtual reality approaches to stroke rehabilitation. Curr Opin Neurol. 2014;27:631-6.

10. Arias P, Robles-García V, Sanmartín G, Flores J, Cudeiro J Virtual reality as a tool for evaluation of repetitive rhythmic movements in the elderly and Parkinson's disease patients Ross O, editor PLoS ONE 2012;7:e30021.

11. Antón D, Goñi A, Illarramendi A. Exercise recognition for Kinect-based telerehabilitation. Methods Inf Med Schattauer GmbH. 2015;54:145-55

12. van Dooren M, Visch V, Spijkerman R, Goossens R, Hendriks V. Personalization in game Design for Healthcare: a literature review on its definitions and effects. IJSG. 2016;3:3-25.

13. Omelina L, Jansen B, Bonnechère B, Van Sint Jan S, Cornelis J. Serious games for physical rehabilitation: Designing highly configurable and adaptable games. Proceedings. Ninth international conference on disability, virtual reality and associated technologies. Laval. 2012. pp. 195-201.

14. van Diest M, Stegenga J, Wörtche HJ, Postema K, Verkerke GJ, Lamoth CJC. Suitability of Kinect for measuring whole body movement patterns during exergaming. J Biomech. 2014;47:2925-32.

15. Bonnechère B, Sholukha V, Omelina L, Van Vooren M, Jansen B, Van Sint Jan S. Suitability of functional evaluation embedded in serious game rehabilitation exercises to assess motor development across lifespan. Gait Posture. 2017:57:35-9.

16. Bonnechère B, Sholukha V, Omelina L, Van Sint Jan S, Jansen B. 3D analysis of upper limbs motion during rehabilitation exercises using the Kinect ${ }^{\text {TM }}$ sensor: development, laboratory validation and clinical application. Sensors. 2018;18:2216.

17. Pandolfo M. Friedreich ataxia: detection of GAA repeat expansions and frataxin point mutations. Methods Mol Med. 2006:126:197-216.

18. Schmitz-Hübsch T, Coudert M, Bauer P, Giunti P, Globas C, Baliko L, et al. Spinocerebellar ataxia types 1, 2, 3, and 6: disease severity and nonataxia symptoms. Neurology. 2008;71:982-9.

19. Tanquy Melac A, Mariotti C, Filipovic Pierucci A, Giunti P, Arpa J, Boesch S, et al. Friedreich and dominant ataxias: quantitative differences in cerebellar dysfunction measurements. J. Neurol. Neurosurg. Psychiatr. 2017::jnnp2017-316964

20. Bonnechère B, Jansen B, Omelina L, Sholukha V, Van Sint Jan S. Validation of the balance Board for Clinical Evaluation Balance during serious gaming rehabilitation exercices. J E Health. 2016;22(9):709-17.

21. Trewartha KM, Garcia A, Wolpert DM, Flanagan JR. Fast but fleeting: adaptive motor learning processes associated with aging and cognitive decline. J Neurosci. 2014;34:13411-21.

22. Teulier C, Lee DK, Ulrich BD. Early gait development in human infants: plasticity and clinical applications. Brumley MR, editor. Dev Psychobiol Wiley-Blackwell; 2015;57:447-458.

23. Hwang S, Tsai C-Y, Koontz AM. Feasibility study of using a Microsoft Kinect for virtual coaching of wheelchair transfer techniques. Biomed Tech (Berl). 2017:62:307-13

Ready to submit your research? Choose BMC and benefit from:

- fast, convenient online submission

- thorough peer review by experienced researchers in your field

- rapid publication on acceptance

- support for research data, including large and complex data types

- gold Open Access which fosters wider collaboration and increased citations

- maximum visibility for your research: over $100 \mathrm{M}$ website views per year

At $\mathrm{BMC}$, research is always in progress.

Learn more biomedcentral.com/submissions 